\title{
Study on the Monitoring and Intervention of Network Public Opinions of
}

\section{Food Safety}

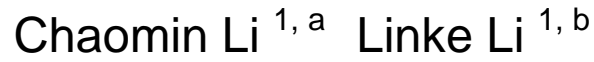 \\ ${ }^{1}$ Luohe Medical College, Luohe City, Henan Province, China, 462000 \\ ${ }^{a}$ email \\ ${ }^{\mathrm{b}}$ email
}

Keywords: Food Safety, Internet Public Opinions, Monitor, Replied Measures

\begin{abstract}
In recent years, with the development of internet, regulations of food safety network public opinions have gradually attracted the attention of government departments. This paper first interprets the connotation of monitoring of food safety network public opinions, and then puts forward three key emphases the food safety network public opinions need to deal with: establishing work system to deal with the food safety network public opinions, doing a good job in daily work of dealing with the food safety network public opinions, obeying the replied procedures of the emergencies of food safety network public opinions.
\end{abstract}

\section{Introduction}

In recent years, the frequent exposure of food safety incidents continues to provoke the public's nerves. Network public opinions spread like a storm, we can see the public concern about food safety, the dissatisfaction with security status, the condemnation of relative enterprises, and the criticism of the management departments. Free discussions are conducive to improve food safety, but some one-sided and extreme views which lack scientific basis easily lead to panic, resulting in social disruption and economic loss. In the face of the serious work of defending food safety, the government faces with huge challenge.

\section{The Connotation of Network Public Opinion Monitoring of Food Safety}

Food safety incidents refer to major accidents which have serious harmful effects or have potentially serious harmful effects on human health and safety. The network public opinions of food safety refer to the open or private beliefs, attitudes, cognition, opinions and emotions and other comprehensive performances formed according to the food safety events within a certain time-space range. Dealing with the network public opinions of food safety refers to that relevant departments unite social forces to resolve the practical problems, to resolve conflicts and crises caused by food safety incident, to eliminate adverse effects, and to guide public opinions turn to a positive side through information dissemination and interaction.

Food safety issues are of high attention and high participation, and the individual events are likely to trigger a wave of public opinions. Due to the net citizens' judgments and social responsibilities are different, the information may be misrepresented and distorted. It will greatly increase the job difficulties of food safety supervision departments, reduce consumers' confidence, lead to huge economic losses of food production, processing, sales and other industries, and even 
affect social harmony and stability. Strengthening monitoring network public opinions of food safety is helpful for relevant departments to deal with risk information, to solve the problems of food safety, to do a good jib of supervision; It is helpful for governments to improve monitoring system of food safety network public opinions, to establish a rapid response system and guide mechanism, to stabilize adverse negative information timely; It is helpful to promote awareness of network security, to promote the virtuous development of the network communication, and to create a healthy and harmonious network environment.

\section{The Establishment of Work System of Dealing with Food Safety Network Public Opinions}

Main requirements of work system of dealing with food safety network public opinions. The government is the main body of the work of dealing with food safety network public opinions, and monitoring public opinions becomes an important work to deal with public opinions. First, it should make public opinion monitoring become a normal work of the relevant government departments, set operating mechanisms to monitor and analyze network public opinions, ascertain specialized personnel, equip technical equipment, guarantee the necessary funds, complete the information gathering, technical analysis, interview and survey, consultation and negotiation, opinions and suggestions of the network public opinions. Second, professional teams of public opinion monitoring should master the skills of computer and network knowledge, understand the national policies on food safety, be familiar with relevant laws, regulations and standard systems, master journalism and communication, information science, statistics, public management science, economics and other disciplines knowledge, possess perfect knowledge and skills of food safety public opinions, be familiar with the structure pattern of public opinions and contradictions, and have a wide scope of knowledge and language skills.

Information sources and information ranges of food safety network public opinions. Information sources of food safety network public opinions can be divided into three blocks: traditional media on the internet, internet speech, mobile phone and network community. Traditional media on the internet refers to network reporting platforms designed by a kind of traditional media such as CCTV, Central People's Broadcasting Station, People's Daily and so on. Internet speeches include special topics of network news and post news, network forum, blog, space and video podcast, network interviews and network survey, rights protection website and so on. Mobile and network communities mainly include social networking sites such as mobile phones, micro-blog, QQ and MSN and other chat tools. In the above information sources, the main scope of network public opinions monitoring of includes: related policy decisions, views of events, views and opinions, food safety incidents and emergency situations, some foreign food safety policies, laws and related food safety emergencies.

Acquisition of network public opinions of food safety. The monitoring of network public opinions of food safety needs to start from a lot of information sources, to find the contents of network public opinions related to food safety. Generally speaking, there are three main ways to get public opinions. First is using Baidu, Google, Sogou and other popular search engines to carry on language retrieval for keywords. Two is browsing web pages and forums in real time through setting public opinion observer positions. Three is working with professional software companies, developing software to monitor and analyze network public opinions of food safety, and capturing network public opinion information in the first time. Compared with other approaches, the third one can greatly improve the accuracy and efficiency of public opinion monitoring, but it needs high costs.. 


\section{Daily work of Network Public Opinions Monitoring of Food Safety}

The monitoring work of food safety network public opinions should be taken as the normal daily work of the relevant government departments. Public opinions observers obtain the relevant food safety network public opinions on the major network platform timely by using above various technical means. Observers first quickly occupy the vast amounts of information, and then identify its sources, authenticity, and points. Through the above analysis, they determine the potential values of public opinion events, exclude false public opinions, and exclude the interference of false information to the real public opinions in the vast amount of information. Then making preliminary judgments to the influence of the public opinion information according to their experience. If the public opinion information has greater impacts or may cause other serious consequences, they should start emergency response procedures of food safety network public opinions. If the public opinion information has little effects, observers should make basic records and simple information analysis.

Public opinion observers should summarize the daily supervision work in the form of daily report, monthly report, semi annual report, annual report and so on, and then come to the scientific monitoring conclusion through the analysis of the data from different time periods and different regions at home and aboard. These summaries are helpful for regulatory agencies to understand peoples' focus, grasp occurrence regularity of public opinions, make work decisions, and adjust the direction of work in the supervision work; or to provide the corresponding research institutions and social public a certain source of information.

\section{Response Procedures of Emergencies of Food Safety Network Public Opinion}

Collection and collation of public opinions. After reviewing, summarizing and analyzing the various important and sensitive information they have collected, and obtaining the emergencies of food safety public opinions which have greater impacts, observers should share relevant information and report the information to superior department and request instructions. This work requires observers to be active, timely and efficient, to ensure the relevant departments get food safety public opinion information the first time, and to provide important information support for the effective implementation of dealing with food safety public opinions.

Analysis and judgment of public opinions. When observing food safety public emergencies or receiving the report from the food safety public emergencies, food safety related regulatory agencies should immediately make qualitative verification and quantitative statistical analysis of collected public opinion information, and judge the authenticity, cause development, data trend and influence degree and other important information of public opinions, and submit it to the superior monitoring unit, and start the corresponding response procedures of food safety public emergencies.

Intervention and disposal of public opinions. After the discovery of major information of public opinions, the relevant regulatory authorities need to have clear responsibilities, intervene and deal with emergencies in the first time. After the initial discovery of information of public opinions, public opinion observers should use their identities to eliminate doubts and confusions, conciliate conflicts while sharing and reporting information of public opinions, and actively guide the public opinions into the right direction. After starting the corresponding response procedures food safety network public opinion emergencies, each supervision departments should set up working teams to deal with public opinions: the leading group, integrated group, data technology group, news media liaison group, experts and crisis public relations group and other basic units. Leading group is in 
charge of the leadership, organization, coordination of the emergency handling of the accidents, issues handling instructions of emergencies. Integrated team is responsible for the implementation of the arrangements for the deployment, and organize the implementation of emergency management. Data technology group is responsible for the analysis and processing of public opinion information, draw the conclusions, and put forward advice and suggestions in the first time. Media contact group is in charge of doing a good job with the public communication, and cooperating with the media to release news and survey interviews and other work, release the of disposal progress of food safety network public opinions. In the expert and crisis public relations group, the former is responsible for providing the corresponding technical advice and answering questions for the government, the latter is mainly responsible for putting forward the scientific and effective emergency responses during the whole process of dealing with public opinions. Various departments and groups strengthen the linkage and collaborate together to respond to development of public opinions.

\section{Conclusion}

Results of emergencies of food safety network public opinions can be large or small. For food safety supervision departments, each step and decision is very important after the emergence of the relevant public opinion events. The response of the network public opinion emergency of food safety likes a "battle", it must be accurate and without accidents, a small detail or a wrong choice may lead to events turn into the opposite direction. Therefore, it is necessary to seriously treat the events of public opinion responses. After finishing the work of dealing with network public opinions of food safety, the relevant departments should promptly make a summary of the work, investigate and handle those who are ineffective in the supervision. For the individuals and organizations who spread malicious rumors, alarmist, said frightening things, deliberately destructed the social harmony and stability must be resolutely crack down, strictly investigated the corresponding legal responsibilities.

\section{References:}

[1] Liu Wen, Li Qiang. Research on monitoring and intervention of food safety network public opinion [J]. China Science and Technology Forum, 2012,07:44-49.

[2] Cao Xia. The practical thinking of network public opinion monitoring under the perspective of food safety [J]. Modern Business, 2012,26:155-156.

[3] Zhu Yunlong, Analysis of monitoring technology system of China's food safety network public opinion [J]. Modern Communication, 2013,04:55-56.

[4] Zhu Yunlong. Guidance to deal with food safety network public opinion [J]. Southeast Communication, 2013,05:43-44.

[5] Zhu Yunlong, Monitoring and guidance of the status quo of China's food safety network public opinion [J]. Young Reporter, 2013,17:14-15.

[6] Pan Qi, Wang Jun, Ren Shugang. Monitoring and response of food safety network public opinion in Ningbo City [J]. Anhui Agricultural Sciences, 2014,32:11504-11505. 